\title{
Nitidine chloride inhibits hepatic cancer growth via modulation of multiple signaling pathways
}

\author{
Jiumao Lin ${ }^{1,2}$, Aling Shen ${ }^{1,2}$, Hongwei Chen ${ }^{1,2}$, Jun Liao ${ }^{3}$, Teng X ${ }^{1,2}$, Liya Liu ${ }^{1,2}$, Jing Lin ${ }^{1,2}$ and Jun Peng ${ }^{1,2^{*}}$
}

\begin{abstract}
Background: The development of hepatic cancer is tightly regulated by multiple intracellular signaling pathways. Therefore, most currently-used anti-tumor agents, which typically target single intracellular pathway, might not always be therapeutically effective. Additionally, long-term use of these agents probably generates drug resistance and unacceptable adverse effects. These problems increase the necessity for the development of new chemotherapeutic approaches. Nitidine chloride (NC), a natural benzophenanthridine alkaloid, has been shown to inhibit cancer growth via induction of cell apoptosis and suppression of cancer angiogenesis. But the precise mechanisms of its tumorcidal activity are not well understood.
\end{abstract}

Methods: To further elucidate the precise mechanisms of its anti-tumor activity, using a hepatic cancer mouse xenograft model, the human hepatic cancer cell lines (HepG2, HCCLM3, Huh7), and umbilical vein endothelial cells (HUVEC), here we evaluate the effect of NC on tumor growth in vivo and in vitro and investigated the underlying molecular mechanisms.

Results: We found that NC treatment resulted in significant decrease in tumor volume and tumor weight respectively, but didn't affect body weight changes. Additionally, NC treatment dose- and time-dependently reduced the cell viability of all three hepatic cell lines. Moreover, NC suppressed the activation of STAT3, ERK and SHH pathways; and altered the expression of critical target genes including Bcl-2, Bax, Cyclin D1, CDK4, VEGF-A and VEGFR2. These molecular effects resulted in the promotion of apoptosis, inhibition of cell proliferation and tumor angiogenesis.

Conclusions: Our findings suggest that NC possesses a broad range of anti-cancer activities due to its ability to affect multiple intracellular targets, suggesting that NC could be a novel multi-potent therapeutic agent for the treatment of hepatic cancer and other cancers.

Keywords: Nitidine chloride, Hepatic cancer, HepG2 cells, Signaling transduction pathways, Cellular proliferation and apoptosis, Tumor angiogenesis

\section{Background}

Primary hepatic cancer or liver cancer is the sixth most commom cancer globally and the second leading cause of cancer-related death [1-5]. The most frequent hepatic cancer is hepatocellular carcinoma (HCC), accounting for approximately $75 \%$ of all primary liver cancers. Another type of liver cancer is hepatoblastoma (HBL), which is specifically formed by immature liver cells and primarily develops in children. To date, chemotherapy remains one

\footnotetext{
* Correspondence: pjunlab@hotmail.com

'Academy of Integrative Medicine Biomedical Research Center, Fujian University of Traditional Chinese Medicine, Fuzhou, Fujian 350122, China ${ }^{2}$ Fujian Key Laboratory of Integrative Medicine on Geriatrics, Fujian University of Traditional Chinese Medicine, Fuzhou, Fujian 350122, China

Full list of author information is available at the end of the article
}

of the major non-surgical therapeutic approaches for patients with advanced hepatic cancer [6]. However, due to drug resistance, systemic chemotherapy produces a disappointing low response rate, ranging between 10\%-15\% [7]. Moreover, many currently used anti-cancer agents have potent cytotoxic effects in normal cells [8]. These problems limit the effectiveness of current HCC chemotherapy, thus increasing the necessity for the development of new chemotherapeutic approaches.

The mechanisms underlying pathogenesis and development of HCC are complex and heterogeneous, involving multiple cellular signaling pathways including signal transducer and activator of transcription 3 (STAT3), Sonic Hedgehog $(\mathrm{SHH})$ and extracellular regulated protein 
kinases (ERK). STAT3 plays an essential role in cell survival, proliferation and angiogenesis [9]. After activation via phosphorylation, STAT3 proteins in the cytoplasm dimerize and translocate to the nucleus where they regulate the expression of critical genes involved in cancer progression. Constitutive activation of STAT3 is strongly associated with cancer development and commonly suggests a poor prognosis $[10,11]$. Aberrant activation of SHH is highly correlated with various human cancers [12-14]. SHH signaling activation is initiated at the cell surface by binding of SHH ligand to the transmembrane receptor Patched (Ptc), resulting in the release of Ptc-mediated suppression of Smoothened (Smo). Smo subsequently activates the Gli family of transcription factors that regulate the expression of various HH target genes [15-17]. Extracellularsignal regulated kinase (ERK) signaling is one of the major cell-survival and proliferation pathways. As a major subfamily member of Mitogen-activated protein kinases (MAPKs), activation of ERK is regulated by a central three-tiered kinase core consisting of MAPK kinase kinase (e.g., Raf), MAPK kinase (e.g., MEK), and MAPK (e.g., ERK); wherein Raf phosphorylates MEK which in turn phosphorylates and activates ERK [18]. By altering the levels and activities of transcription factors, activation of ERK pathway regulates the expression of various genes mediating cell apoptosis, proliferation and angiogenesis $[19,20]$. These molecular pathways described above modulate the expression of key genes involved in the regulation of cell proliferation, apoptosis, and angiogenesis and are participants in the processes of induction, progression, and metastasis of hepatic cancer. Thus, each serves as a potential target for novel chemotherapeutics.

Natural products have received recent interest in discovery of novel anti-cancer therapeutic agents as they have relatively few side effects and have long been used as alternative remedies for a variety of diseases including cancer $[21,22]$. Therefore, identifying naturally occurring agents is a promising approach for anticancer treatment. Nitidine chloride, a natural benzophenanthridine alkaloid, is a major active compound present in a wellknown traditional Chinese medicinal herb Zanthoxylum nitidum (Roxb) DC. Previous studies found that NC has antifungal, anti-inflammatory and analgesic activities $[23,24]$. Recently it has been shown that NC inhibits the growth of many human cancer cells via induction of cell apoptosis [25]. Moreover, Chen et al. reported that NC can suppress gastric cancer angiogenesis by inhibition of STAT3 pathway [26], and we previously reported that the $\mathrm{NC}$ is able to inhibit hepoatocellular carcinoma growth via modulation of JAK1/STAT3 pathway [27]. In order to further elucidate the mechanism of tumorcidal activity of $\mathrm{NC}$, in the present study we evaluated its effect on hepatic cancer growth in vivo and in vitro, and investigated the underlying molecular mechanisms.

\section{Methods}

\section{Materials and reagents}

Nitidine Chloride (NC, purity $>98 \%$ ) was provided from Institute of Sichuan Xianxin Biochemical Technology (Sichuan, China). Matrigel was provided by Becton Dickinson (San Jose, CA, USA). Roswell Park Memorial Institute Medium 1640 (RPMI 1640), Dulbecco's modified Eagle's medium (DMEM), fetal bovine serum (FBS), penicillin-streptomycin, trypsin-EDTA, 5,5,6,6'-tetrachloro1,1,3,3'-tetraethyl-benzimidazol-carbocyanine iodide (JC-1), were purchased from Invitrogen (Grand Island, NY, USA). The In Vitro Angiogenesis Assay Kit was purchased from Millipore (Billerica, MA, USA). A fluorescein isothiocyanate (FITC)-conjugated annexin V apoptosis detection kit was provided by Becton Dickinson (San Jose, CA, USA). TUNEL assay kit (TumorTACS in situ) was purchased from R\&D Systems (Minneapolis, MN, USA). All antibodies were purchased from Cell Signaling Technology (Beverly, MA, USA). BCA Protein Assay Kit was purchased from Tiangen Biotech Co., Ltd. (Beijing, China). Cignal STAT3 Reporter (luc) Kit was obtained from SABiosciences, QIAGEN company (Hilden, Germany). All other chemicals, unless otherwise stated, were obtained from Sigma-Aldrich (St. Louis, MO, USA).

\section{Cell culture}

Human hepatic cancer cell lines (HepG2, HCCLM3 and Huh7) and human umbilical vein endothelial cells (HUVECs) were purchased from Xiangya Cell Center (Hunan, China). HepG2 cells and HUVECs were grown in DMEM and RPMI 1640, respectively. Both DMEM and RPMI 1640 were supplemented with $10 \%$ (v/v) FBS, $100 \mathrm{units} / \mathrm{ml}$ penicillin, and $100 \mu \mathrm{g} / \mathrm{ml}$ streptomycin.

\section{Animals}

Male BALB/C athymic nude mice (with an initial body weight of 20-22 g) were obtained from Shanghai SLAC Laboratory Animal Co., Ltd. (Shanghai, China) and housed under pathogen-free conditions with controlled temperature $\left(22^{\circ} \mathrm{C}\right)$, humidity, and a 12 hour light/dark cycle. Food and water were given ad libitum throughout the experiment. All animal treatments were performed strictly in accordance with international ethical guidelines and the National Institutes of Health Guide concerning the Care and Use of Laboratory Animals. The experiments were approved by the Institutional Animal Care and Use Committee of Fujian University of Traditional Chinese Medicine.

\section{In vivo nude mice xenograft study}

Hepatic cancer xenograft mice were produced with HepG2 cells. The cells were grown in culture and then detached by trypsinization, washed, and resuspended in serum-free DMEM. Resuspended cells $\left(5 \times 10^{6}\right)$ mixed 
with Matrigel (1:1) were subcutaneously injected into the right flank of mice to initiate tumor growth. At 5 days following xenograft implantation (tumor size approximately $3 \mathrm{~mm}$ in diameter), mice were randomized into two groups $(n=10)$ and treated with $4.5 \mathrm{mg} / \mathrm{kg}$ of $\mathrm{NC}$ (dissolved in saline) or saline daily by intraperitoneal injection, 6 days a week for 18 days. Body weight and tumor size were measured. Tumor size was determined by measuring the major (L) and minor (W) diameter with a caliper. The tumor volume was calculated according to the following formula: tumor volume $=\pi / 6 \times \mathrm{L} \times \mathrm{W}^{2}$. At the end of the experiment, the animals were anaesthetized and tumors were excised and weighed.

\section{Cell viability evaluation by MTT assay}

$\mathrm{NC}$ was dissolved in DMSO and diluted to working concentrations with culture medium. The final concentration of DMSO in the medium for all cell-based experiments was $0.1 \%$. Cells (HepG2, HCCLM3, Huh7 cells or HUVECs) were seeded into 96-well plates at a density of $1.0 \times 10^{4}$ cells/well in $0.1 \mathrm{ml}$ medium. $24 \mathrm{~h}$ later, cells were treated with various concentrations of $\mathrm{NC}$ for different time periods. After NC treatment, $10 \mu \mathrm{MTT}(5 \mathrm{mg} / \mathrm{ml}$ in phosphate buffered saline (PBS)) were added to each well, and the samples were incubated for an additional $4 \mathrm{~h}$ at $37^{\circ} \mathrm{C}$. The purple-blue MTT formazan precipitate was dissolved in $100 \mu \mathrm{l}$ DMSO. Absorbance was measured at $570 \mathrm{~nm}$ using an ELISA reader (BioTek, Model EXL800, USA).

\section{Colony formation assay}

HepG2 cells from different treated groups were seeded in 6-well plates with a density of 200 cells per well for 7 days. The medium was discarded and each well was washed twice with PBS carefully. The colonies were fixed in methanol for $20 \mathrm{~min}$ and then stained with Giemsa staining solution. The number of colonies with $\geq 50$ cells was counted and colony forming efficiency was calculated (Percentage of colonies $=$ Number of colonies formed/Number of cells inoculated $\times 100 \%$ ).

\section{Cell cycle analysis}

Cell cycle analysis was carried out by flow cytometry using FACS analysis with propidium iodide (PI) staining. HepG2 cells were treated with various concentrations of $\mathrm{NC}$ for $24 \mathrm{~h}$, harvested, adjusted to a concentration of $1 \times 10^{6}$ cells $/ \mathrm{ml}$, and fixed in $70 \%$ ethanol at $4^{\circ} \mathrm{C}$ overnight. The fixed cells were washed twice with cold PBS, and incubated for $30 \mathrm{~min}$ with RNase $(8 \mu \mathrm{g} / \mathrm{ml})$ and PI $(10 \mu \mathrm{g} / \mathrm{ml})$. The fluorescent signal was detected through the FL2 channel and the proportion of DNA in different phases was analyzed using ModfitLT Version 3.0 (Verity Software House, Topsham).

\section{Apoptosis detection in HepG2 cells by flow cytometry analysis with annexin V/PI staining}

After incubation with various concentrations of $\mathrm{NC}$, apoptosis of HepG2 cells were determined by flow cytometry using a fluorescence-activated cell sorting (FACS) caliber (Becton Dickinson, CA, USA) and Annexin V-fluorescein isothiocyanate (FITC)/propidium iodide (PI) kit (Becton Dickinson). Staining was performed according to the manufacturer's instructions. The percentage of cells in early apoptosis was calculated by Annexin V-positivity and PI-negativity, and the percentage of cells in late apoptosis was calculated by Annexin V-positivity and PI-positivity.

Measurement of mitochonrial membrane potential $(\Delta \psi \mathrm{m})$ by flow cytometry analysis with JC- 1 staining

JC-1 is a cationic dye that exhibits potential mitochondria-dependent accumulation, indicated by a fluorescence emission shift from green to red, which thus can be used as an indicator of mitochondrial potential. In this experiment, $1 \times 10^{6}$ treated HepG2 cells were resuspended after trypsinization in $1 \mathrm{ml}$ of medium and incubated with $10 \mu \mathrm{g} / \mathrm{ml}$ of $\mathrm{JC}-1$ (Invitrogen) at $37^{\circ} \mathrm{C}$, $5 \% \mathrm{CO}_{2}$, for $30 \mathrm{~min}$. Both red and green fluorescence emissions were analyzed by flow cytometry after JC-1 staining.

\section{Apoptosis detection in hepatic tumor tissues by TUNEL staining}

Six tumors were randomly selected from NC-treatment or control groups. Tumor tissues were fixed in $10 \%$ formaldehyde for $12 \mathrm{~h}$, paraffin-embedded and then sectioned into 4- $\mu$ m-thick slides. Samples were analyzed by TUNEL staining using TumorTACS in situ kit (R\&D Systems). Apoptotic cells were counted as DAB-positive cells (brown stained) at five arbitrarily selected microscopic fields at a magnification of $400 \times$. TUNEL-positive cells were counted as a percentage of the total cells.

Immunohistochemistical analysis of hepatic tumor tissues Six tumors were randomly selected from NC-treatment or control groups. Tumor tissues were fixed in 10\% formaldehyde for $12 \mathrm{~h}$, paraffin-embedded, sectioned, and placed on slides. The slides were subjected to antigen retrieval and endogenous peroxidase activity was quenched with hydrogen peroxide. Non-specific binding was blocked with normal serum in PBS (0.1\% Tween 20). Rabbit polyclonal antibodies against Ki-67, CD31, Shh and Gli-1 (all in 1:200 dilution, Santa Cruz Biotechnology) were used to detect the relevant proteins. The binding of the primary antibody was demonstrated with a biotinylated secondary antibody, horseradish peroxidase (HRP)-conjugated streptavidin (Dako), and diamino-benzidine (DAB) as the chromogen. The tissues were counterstained with diluted Harris hematoxylin. After staining, five high- 
power fields (at magnification of $400 \times$ ) were randomly selected in each slide. The proportion of positive cells in each field was determined using the true color multifunctional cell image analysis management system (Image-Pro Plus, Media Cybernetics, USA). To control for nonspecific staining, PBS was used to replace the primary antibody as a negative control.

\section{Tube formation assay of HUVECs}

HUVEC tube formation was examined using the ECMatrix assay kit (Millipore) following the manufacturer's instructions. Briefly, confluent HUVECs were harvested and diluted $\left(1 \times 10^{4}\right.$ cells $)$ in $50 \mu$ l of medium containing various concentrations of NC. The harvested cells were seeded with ECMatrix gel $(1: 1 \mathrm{v} / \mathrm{v})$ into 96 -well plates and incubated for $9 \mathrm{~h}$ at $37^{\circ} \mathrm{C}$. The cells were photographed using phase-contrast inverted microscopy at a magnification of $100 \times$.

\section{RT-PCR analysis}

Total RNA was isolated from tumor tissues (three tumors were randomly selected from NC-treatment or control groups) or HepG2 cells and HUVECs with TriZol Reagent (Invitrogen). Oligo (dT)-primed RNA (1 $\mu \mathrm{g})$ was reverse-transcribed with SuperScript II reverse transcriptase (Promega) according to the manufacturer's instructions. The obtained cDNA was used to determine the mRNA amount of Cyclin D1, CDK4, Bcl-2, Bax, SHH, Gli-1, VEGF and VEGFR2 by PCR with Taq DNA polymerase (Fermentas). GAPDH was used as an internal control. Samples were analyzed by gel electrophoresis (1.5\% agarose). The DNA bands were examined using a Gel Documentation System (BioRad, Model Gel Doc XR+, USA).

\section{Western blotting analysis}

Three tumors were randomly selected from NCtreatment or control groups. Tumor tissues were homogenized in nondenaturing lysis buffer and centrifuged at $14,000 \times \mathrm{g}$ for $15 \mathrm{~min}$. Protein concentrations of the clarified supernatants were determined by BCA protein assay. HepG2 cells or HUVECs $\left(2.5 \times 10^{5}\right)$ in $5 \mathrm{ml}$ medium were seeded into $25 \mathrm{~cm}^{2}$ flasks and treated with the indicated concentrations of $\mathrm{NC}$ for $24 \mathrm{~h}$. Treated cells were lysed in mammalian cell lysis buffer (M-PER, Thermo Scientific, Rockford, IL, USA) containing protease (EMD Biosciences) and phosphatase inhibitor (Sigma-Aldrich) cocktails, and centrifuged at $14,000 \times \mathrm{g}$ for $15 \mathrm{~min}$. Protein concentrations in cell lysate supernatants were determined by BCA protein assay. Equal amounts of protein from each tumor or cell lysate were resolved on $12 \%$ Tris-glycine gels and transferred onto PVDF membranes. The membranes were blocked for $2 \mathrm{~h}$ with $5 \%$ nonfat dry milk and incubated with the desired primary antibody directed against STAT3, pSTAT3, ERK, pERK, Cyclin D1, CDK4, Bcl-2, Bax, VEGF, VEGFR2 or $\beta$-actin (all in 1:1000 dilutions) overnight at $4^{\circ} \mathrm{C}$. Appropriate HRP-conjugated secondary antibodies with chemiluminescence detection were used to image the antibody-detected proteins.

\section{Luciferase gene reporter assay}

HepG cells were seeded into 96-well plates at a density of $1 \times 10^{4}$ cells/well in $0.1 \mathrm{ml}$ complete DMEM until about $50 \%$ confluency and then continuously cultured in FBSand antibiotics-free medium overnight. Cells were transfected with a mixture of inducible STAT3-responsive firefly luciferase construct and constitutively expressing Renilla luciferase construct using Lipofectamine ${ }^{\mathrm{Tm}}$ LTX with PLUS ${ }^{\mathrm{rm}}$ Reagent. $6 \mathrm{~h}$ after transfection the medium was changed back into DMEM complete with FBS, penicillin and streptomycin. After 24 hours of transfection, cells were treated with various contractions of $\mathrm{NC}$ for $1 \mathrm{~h}$ followed by IL-6 for another $24 \mathrm{~h}$. Cell extracts were prepared and analyzed using Promega Dural Luciferase Reporter Assay System according to the manufacturer's instruction. The measured firefly luciferase activity was normalized to the activity of Renilla luciferase in the same well.

\section{Statistical analysis}

Data were presented as mean $\pm \mathrm{SD}$ for the indicated number of independently performed experiments. Statistical analysis was carried out with Student's $t$-test and ANOVA. Differences with $P<0.05$ were considered to be statistically significant.

\section{Results and discussion}

\section{$\mathrm{NC}$ inhibits hepatic cancer growth in vitro and in vivo}

We evaluated the in vitro anti-cancer effect of $\mathrm{NC}$ by examining the viability of three human hepatic cell lines (HepG2, HCCLM3 and Huh7) using MTT assay. As shown in Figure 1A, treatment with NC dose- and timedependently reduced the viability of all three hepatic cell lines $(P<0.05)$. The in vivo anti-tumor activity of $\mathrm{NC}$ was evaluated by comparing the tumor weight and volume in treated and control HepG2 xenograft mice, while its adverse effects were determined by measuring changes in body weight. As shown in Figure 1B-D, NC treatment resulted in $56.62 \%$ and $36.14 \%$ decrease in tumor volume and tumor weight respectively, as compared to control $(P<0.01)$. However, administration of $\mathrm{NC}$ had no effect on body weight changes during the course of the study (Figure 1E). Taken together, these data suggest that $\mathrm{NC}$ is effective in suppressing liver tumor growth both in vivo and in vitro, without apparent signs of toxicity. 

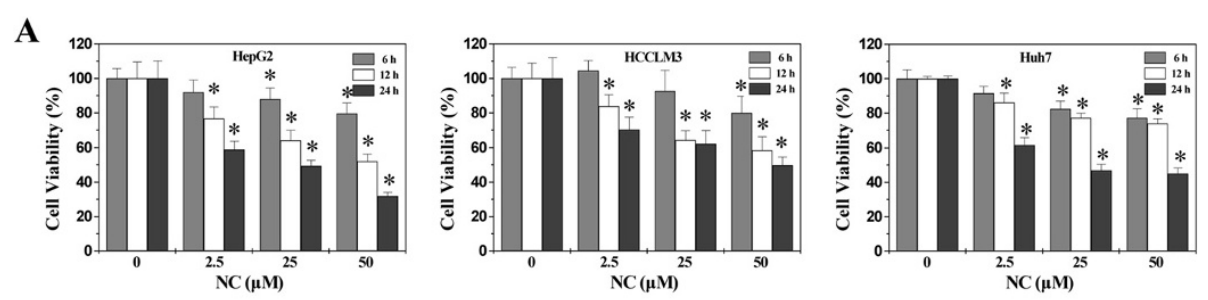

B

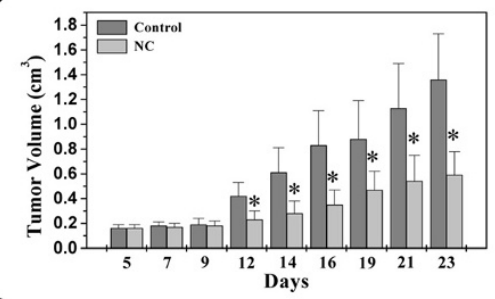

D

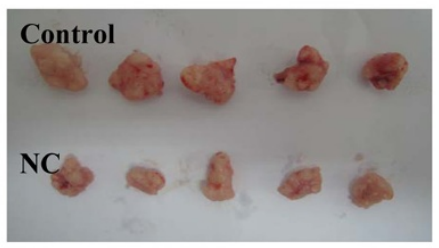

C

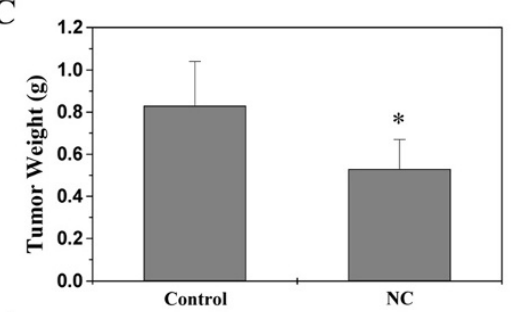

$\mathbf{E}$

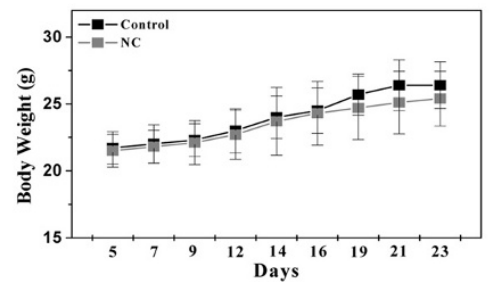

Figure 1 Effect of NC on hepatic cancer growth in vitro and in vivo. (A) Viability of HepG2, HCCLM3 or Huh7 cells was determined by the MTT assay after cells were treated with the different concentrations of $\mathrm{NC}$ for the indicated time periods. ${ }^{*} P<0.05$, versus control cells. (B-E) In vivo study. After tumor development, mice were randomized into two groups $(n=10)$ and treated with $4.5 \mathrm{mg} / \mathrm{kg}$ of NC or saline daily by intraperitoneal injection, 6 days a week for 18 days. Tumor volume (B), tumor weight (C) and body weight (E) were measured. (D) Representative images for tumors. Data shown are averages with S.D. (error bars) from 10 individual mouse in each group. ${ }^{*} P<0.01$, versus controls.
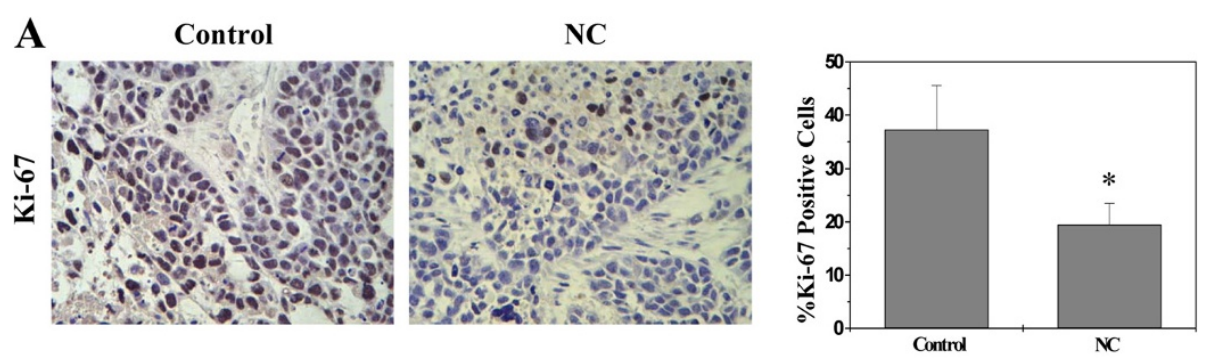

B

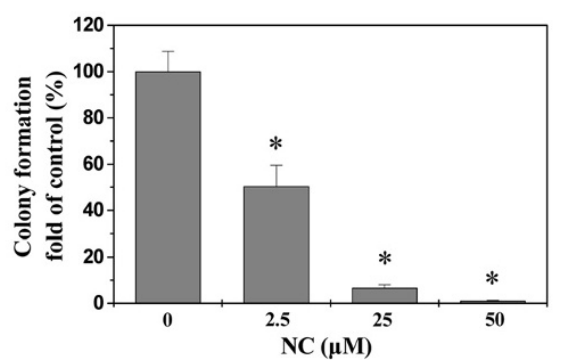

C

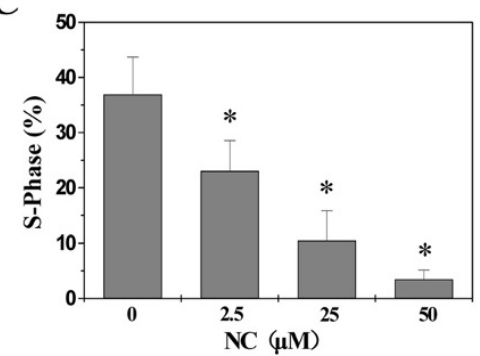

Figure 2 Effect of NC on cell proliferation in hepatic cancer xenograft mice and HepG2 cells. (A) Ki-67 assay in tumor tissues (400 $\times$ ). Data shown are averages with S.D. (error bars) from 6 individual mouse in each group. ${ }^{*} P<0.01$, versus controls. (B) HepG2 cell colony formation assay. The data were normalized to the viability or survival of control cells (100\%, treated with $0.1 \%$ DMSO vehicle). (C) HepG2 cell cycle was analyzed by FACS and the proportion of cells in S-phase was calculated. Data from panels B-D are averages with S.D. (error bars) from at least three independent experiments. ${ }^{*} P<0.01$, versus control cells. 


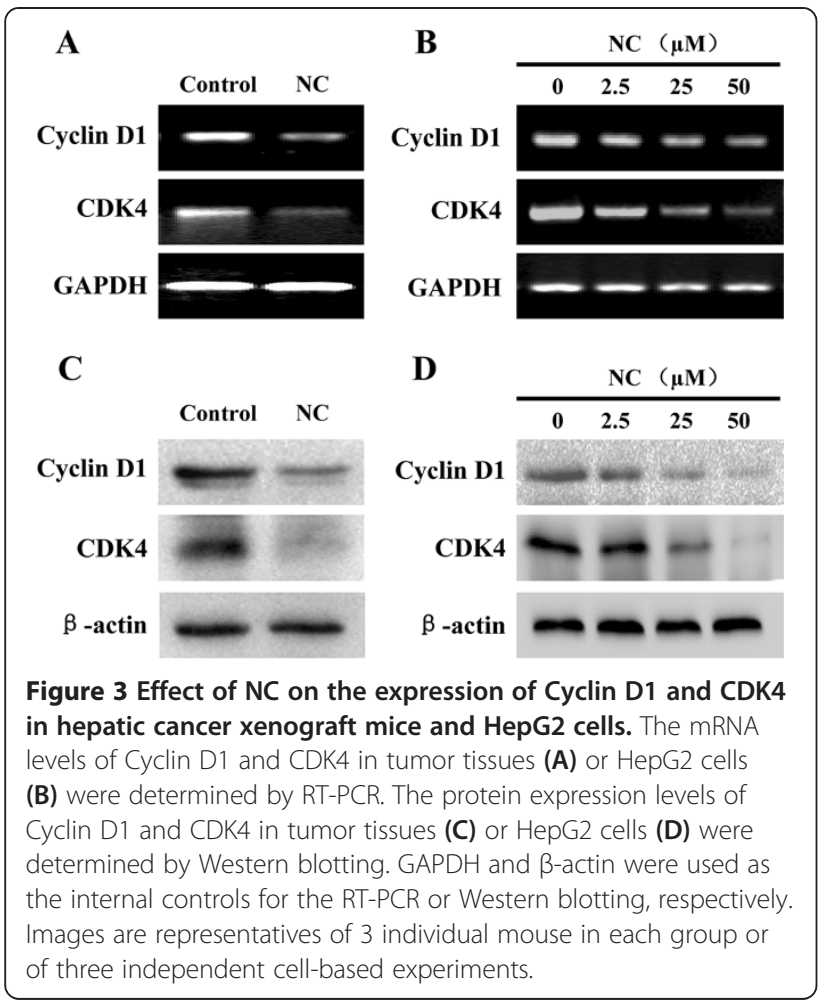

NC inhibits hepatic cancer cell proliferation via G1/S cell cycle arrest

Cancer cells are characterized by an uncontrolled increase in cell proliferation; we therefore determined the proliferative activity of NC. Cell proliferation in tumor tissues was determined via immunohistochemical staining (IHC) for $\mathrm{Ki}-67$, a proliferation marker that is specifically expressed in proliferating cell nuclei. As shown in Figure 2A, the percentage of Ki-67 positive cells in tumor tissues from control and NC-treated xenograft mice was $37.3 \pm 8.3 \%$ and $19.5 \pm 4.0 \%$, respectively $(P<0.01)$. The in vitro proliferation of HepG2 cells was determined by colony formation assay. As shown in Figure 2B, treatment with 2.5, 25 and $50 \mu \mathrm{M}$ of $\mathrm{NC}$ for $24 \mathrm{~h}$ reduced the cell survival rate by $50 \%, 93 \%$ and $99 \%$ compared to untreated control cells $(P<0.01)$. Thus, NC can inhibit hepatic cancer cell proliferation both in vivo and in vitro.

Eukaryotic cell proliferation is primarily regulated by cell cycle. G1/S transition is one of the major cell cycle checkpoints [28], which is responsible for initiation and completion of DNA replication. G1/S progression is strongly regulated by Cyclin D1 that exerts its function via forming an active complex with its CDK major catalytic partners (CDK4/6) [29]. An unchecked or hyperactivated Cyclin D1/CDK4 complex often leads to uncontrolled cell division and malignancy [30-32]. The effect of $\mathrm{NC}$ on the G1 to $\mathrm{S}$ progression in HepG2 cells was investigated via PI staining followed by FACS analysis. We found that the

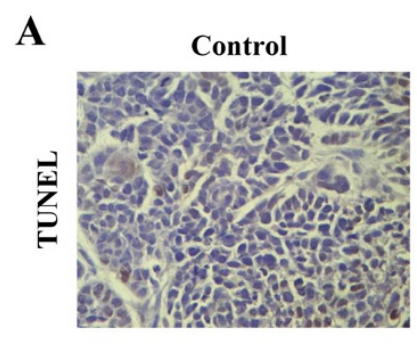

B

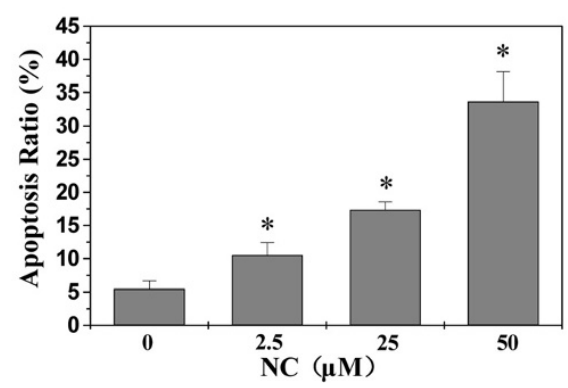

NC
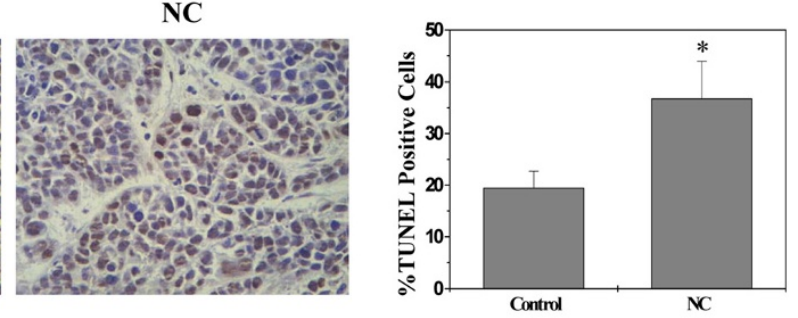

C

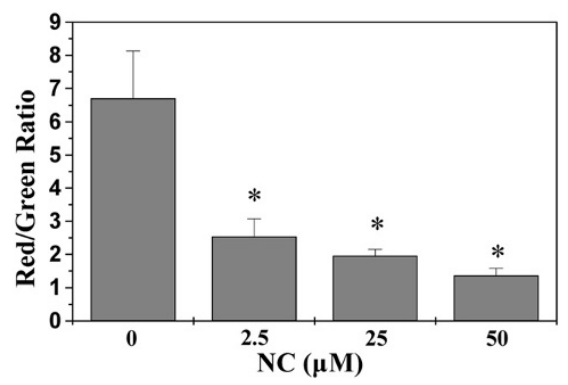

Figure 4 Effect of NC on cell apoptosis in hepatic cancer xenograft mice and HepG2 cells. (A) TUNEL assay in tumor tissues (400 $\times$ ). Data shown are averages with S.D. (error bars) from 6 individual mouse in each group. ${ }^{*} P<0.01$, versus controls. (B) Apoptosis of HepG2 cells were quantification of FACS analysis. (C) Quantitative analysis of the JC-1 red/green fluorescent intensity ratio. The data from C and E shown are averages with S.D. (error bars) from three independent experiments. ${ }^{*} P<0.05$, versus controls. 


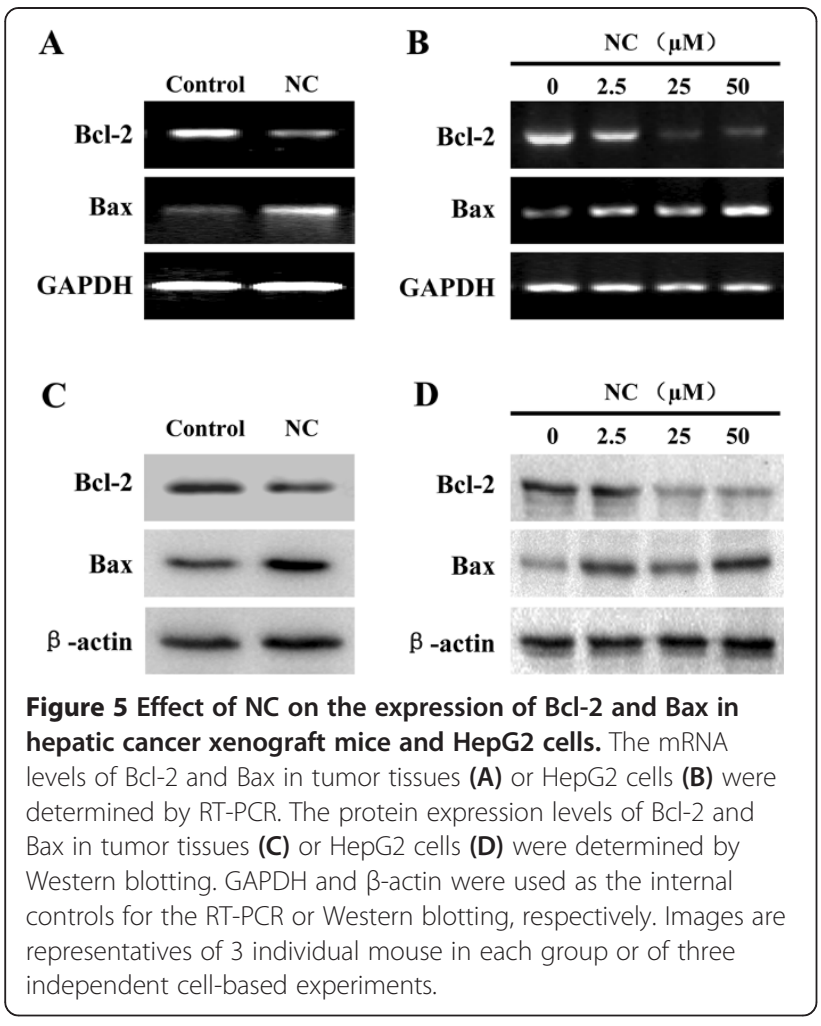

percentage of HepG2 cells in S-phase following NC treatment was decreased in a dose-dependent manner (Figure 2C, $P<0.01$ ). In addition, data from RT-PCR and Western Blot analysis showed that $\mathrm{NC}$ treatment significantly reduced the mRNA and protein levels of proproliferative Cyclin D1 and CKD4 both in hepatic tumor tissues and HepG2 cells (Figure 3). These data together suggest that $\mathrm{NC}$ inhibits hepatic cancer cell proliferation through blockade of G1-S progression and the modulation of the expression of cell cycle-regulatory genes.

\section{$\mathrm{NC}$ induces hepatic cancer cell apoptosis via activation of} the mitochondrion-dependent pathway

Apoptosis is crucial for animal development and tissue homeostasis. Disturbed regulation of this vital process represents a major causative factor in tumorigenesis. Promoting cell apoptosis therefore has been a major focus in the development of anti-cancer therapies. In the present study we evaluated apoptosis in tumors via TUNEL assay; and we found that the percentage of TUNEL-positive cells in tumors from NC-treated mice or controls was $36.8 \pm 7.1 \%$ or $19.5 \pm 3.2 \%$, respectively (Figure $4 \mathrm{~A}, P<0.01$ ), suggesting that $\mathrm{NC}$ promotes cell apoptosis in tumor tissues. The apoptosis of HepG2 cells was determined via Annexin-V/PI staining followed by FACS analysis. As shown in Figure 4B, the percent of cells undergoing either

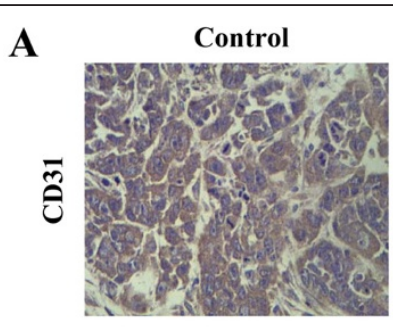

B

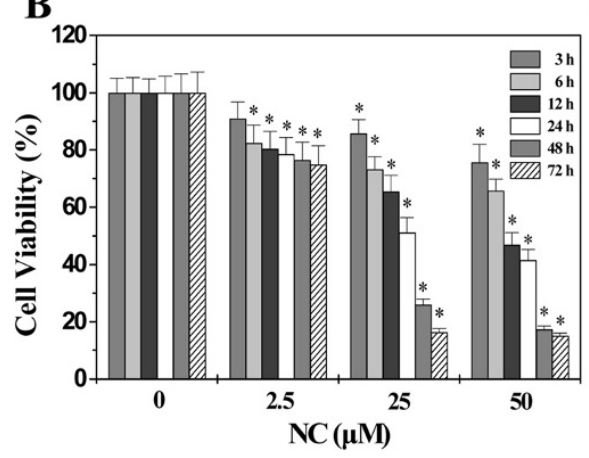

NC
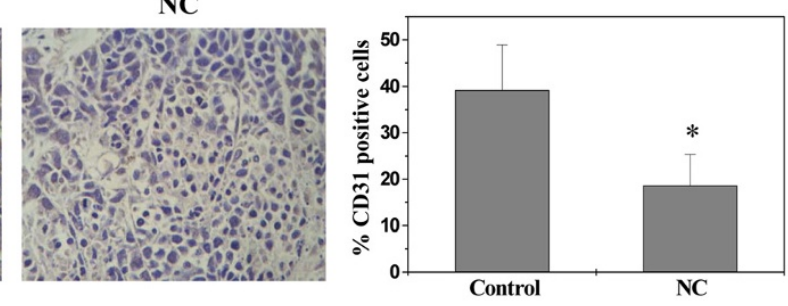

C
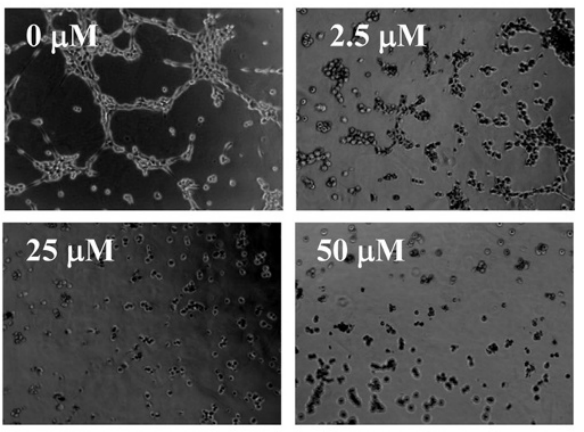

Figure 6 Effect of NC on angiogenesis in hepatic cancer xenograft mice and HUVECs. (A) CD31 assay in tumor tissues (400 X). Data shown are averages with S.D. (error bars) from 6 individual mouse in each group. ${ }^{*} P<0.01$, versus controls. (B) HUVEC's viability was determined by the MTT assay after cells were treated with the various concentrations of NC for the indicated time periods. The data were normalized to the viability of control cells (100\%, treated with $0.1 \%$ DMSO vehicle). Data are averages with S.D. (error bars) from at least three independent experiments. ${ }^{*} P<0.05$, versus controls. (C) Tube formation assay in HUVEc $(100 \times)$. The network-like structures were examined by phase-contrast microscopy. Images are representatives of three independent experiments. 
early or late apoptosis following treatment with $2.5,25$ or $50 \mu \mathrm{M}$ of $\mathrm{NC}$ was $8.50 \%, 17.12 \%$ or $32.19 \%$, whereas the apoptotic rate in untreated control cells was $5.48 \%$ $(P<0.05)$. Thus NC induces hepatic cancer cell apoptosis in vitro in a dose-dependent manner.

The mitochondrion-dependent pathway is the most common apoptotic pathway in vertebrate animal cells. Mitochondrial outer membrane permeabilization (MOMP) is a key commitment step in the induction of cellular apoptosis, since it is the point of convergence for a large variety of intracellular apoptotic signaling pathways leading to the release of many apoptogenic proteins from the mitochondrial intermembrane space. During the process of MOMP, the electrochemical gradient across the mitochondrial membrane collapses. Therefore, the loss of mitochondrial membrane potential is a hallmark for apoptosis. To investigate the mechanism of how NC induced HepG2 cell apoptosis, we used FACS analysis with JC-1 staining to examine the change of mitochondrial membrane potential in NC-treated HepG2 cells. The membrane-permeant JC-1 dye displays potential-dependent accumulation in mitochondria, indicated by a fluorescence emission shift from green $(\sim 525 \mathrm{~nm})$ to red $(\sim 590 \mathrm{~nm})$. Therefore, collapse of mitochondrial potentail during apoptosis is indicated by a decrease in the ratio of red/green fluorescence intensity. As shown in Figure 4C, after treatment with 0, $2.5,25,50 \mu \mathrm{M}$ of $\mathrm{NC}$ the JC-1 red/green fluorescent ratio in HepG2 cells was $6.71 \pm 1.52,2.54 \pm 0.53,1.96 \pm 0.19$, $1.36 \pm 0.22$, respectively, suggesting that NC dose-dependently induces the loss of mitochondrial membrane potential in HepG2 cells.

Bcl-2 family proteins are key regulators of mitochondrion-mediated apoptosis, functioning as either suppressors such as Bcl-2, or promoters such as Bax. MOMP is thought to occur through the formation of pores in the mitochondria by pro-apoptotic Bax-like proteins, which can be inhibited by anti-apoptotic Bcl-2-like members. Therefore, the ratio of active anti- and pro-apoptotic Bcl-2 family members determines the fate of cells, and alteration of the ratio by aberrant expression of these proteins impairs the normal apoptotic program contributing to various apoptosis-related diseases including cancer [33]. Higher Bcl-2 to Bax ratios are commonly found in cancers, which not only confers a survival advantage to the cancer cells but also causes resistance to chemo- and radio-therapies. To further study the mechanism of NC's pro-apoptotic activity, we performed RT-PCR and Western Blotting to respectively examine the mRNA and protein expression of Bcl-2 and Bax. As shown in Figure 5, NC treatment significantly reduced the mRNA and protein expression levels of anti-apoptotic Bcl-2 both in the tumor tissues and HepG2 cells, whereas those of pro-apoptotic Bax were significantly increased after NC treatment, suggesting that NC induces hepatic cancer cell apoptosis both in vivo and in vitro through an increase in the pro-apoptotic Bax/Bcl-2 ratio.

\section{NC inhibits hepatic tumor angiogenesis via suppressing the expression of VEGF-A and VEGFR2}

Formation of new blood vessels via angiogenesis is critical for the development of solid tumors [34-37]. Initially, tumor cells obtain oxygen and nutrients from nearby blood vessels by simple passive diffusion. However, when tumor grows to certain size, oxygen delivery by diffusion is no longer sufficient, which causes tumor cells to induce the sprouting of new blood vessels from pre-existing vasculature, creating a blood supply system within solid tumor that is essential for continuous growth of tumor as well as providing an avenue for hematogenous metastasis $[38,39]$. To determine the effect of NC on angiogenesis in vivo, we performed IHC for the expression of the endothelial cell-specific marker CD31 to examine intratumoral microvessel density (MVD). As shown in Figure 6A, the percentage of CD31-positive cells in NC-treated mice was significantly reduced $(P<0.01)$. The processes of angiogenesis include endothelial cell (EC) proliferation, migration, and alignment into tubular structures. To further

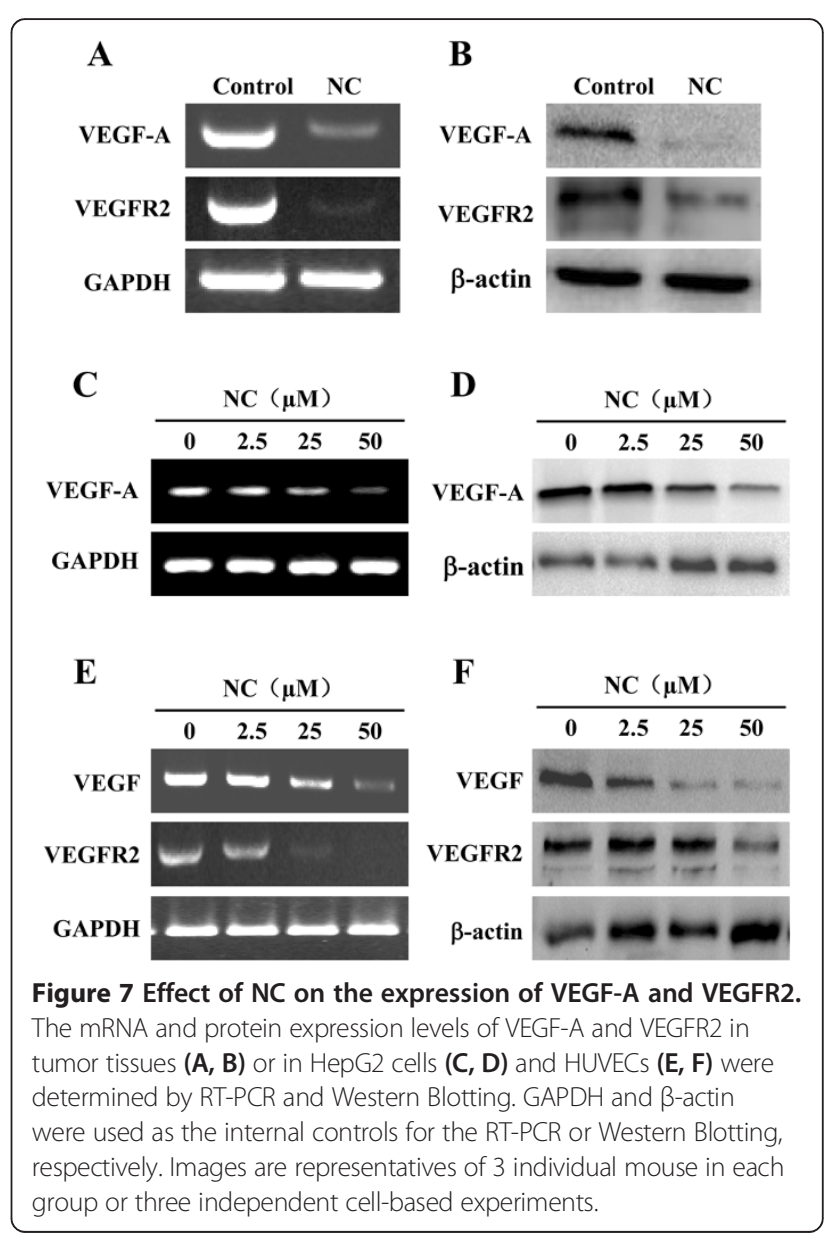




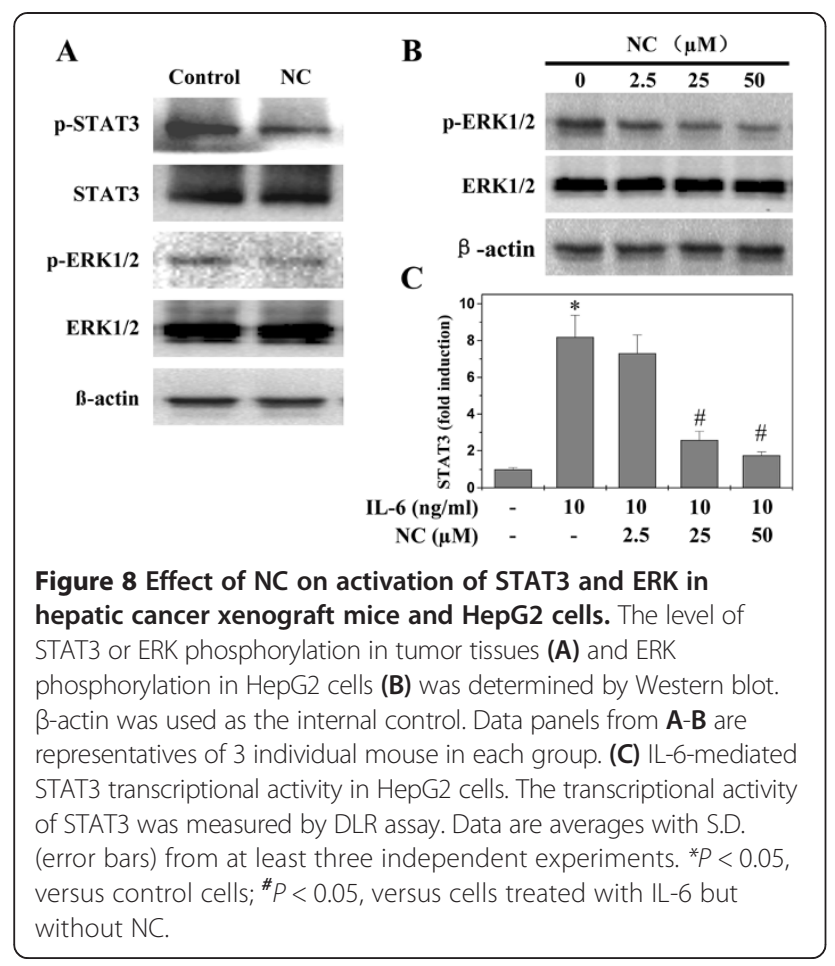

evaluate the anti-angiogenic effect of $\mathrm{NC}$ we modeled each of these processes with HUVECs in vitro. As shown in Figure 6B, NC treatment dose- and time-dependently decreased the proliferation (viability) of HUVECs compared to untreated control cells $(P<0.05)$. Moreover, we examined NC's effect on capillary tube formation of HUVECs using an extracellular matrix, in which cultured ECs rapidly align and form hollow tube-like structures. As shown in Figure 6C, untreated HUVECs formed elongated tube-like structures, whereas $\mathrm{NC}$ treatment resulted in a significant decrease in capillary tube formation. Collectively, these data suggest that inhibition of tumor angiogenesis by NC could have contributed to the inhibition of hepatic cancer growth.

Induction of angiogenesis is mediated by a variety of molecules released by tumor cells [40]. Vascular endothelial growth factor A (VEGF-A) is considered one of the strongest angiogenic stimulators [41]. VEGF-A is highly expressed in a wide variety of human tumors, which has been associated with tumor progression, invasion and metastasis, and poorer survival and prognosis in patients $[42,43]$. VEGF-A is secreted by tumor cells and endothelial cells and functions via paracrine and autocrine signaling pathways. When VEGF-A is secreted, it primarily

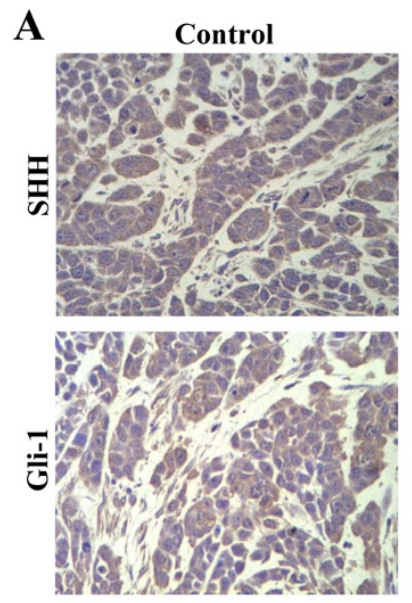

B

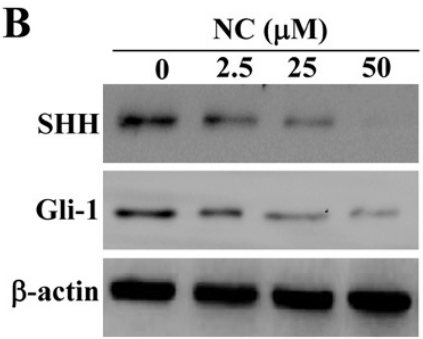

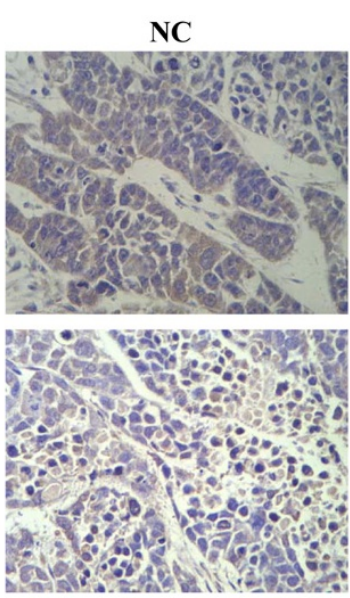

C

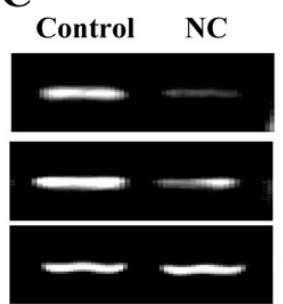

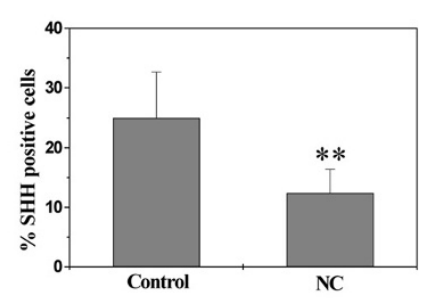

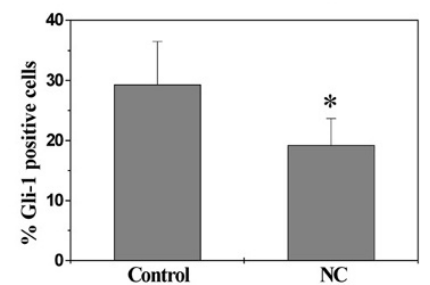

\section{D}

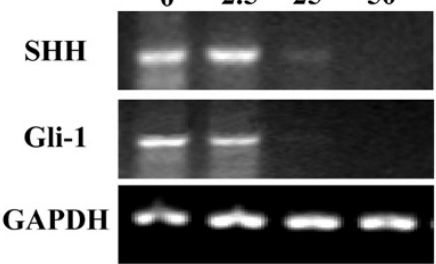

Figure 9 Effect of NC on the activation of SHH pathway in hepatic cancer xenograft mice and HepG2 cells. (A) Tumor tissues were processed for IHC for $\mathrm{SHH}$ and Gli-1. The photographs are representative images taken at a magnification of $400 \times$. Quantification of IHC assay was represented as percentage of positively-stained cells. Data shown are averages with S.D. (error bars) from 6 individual mouse in each group. ${ }^{*} P<0.05 ;{ }^{* *} P<0.01$, versus controls. (B) The protein expression levels of Shh and Gli-1 in HepG2 cells were determined by Western Blotting. $\beta$-actin was used as the internal control. The mRNA levels of SHH and Gli-1 in tumor tissues (C) and HepG2 cells (D) were determined by RT-PCR. GAPDH was used as the internal control. Images of Western Blotting and RT-PCR are representatives of 3 individual mouse in each group. 
binds to specific receptors located on vascular endothelial cells (EC) [44], which in turn triggers a tyrosine kinase signaling cascade that eventually induces angiogenesis $[44,45]$. To explore the mechanism of NC's anti-angiogenic activity, we determined its effect on the expression of VEGF-A and its specific receptor VEGFR2 both in vivo and in vitro. As shown in Figure 7A and B, NC treatment profoundly decreased mRNA and protein levels of VEGF-A and VEGFR2 in hepatic cancer xenograft tumor tissues. Similarly, NC dose-dependently reduced VEGF-A expression in HepG2 cells and HUVECs and also the expression of VEGFR-2 in HUVECs, at both transcriptional and translational levels (Figure 7C-F).

\section{NC suppresses STAT3, ERK and SHH pathways}

Cancer development is tightly regulated by multiple intracellular signaling pathways, including STAT3, ERK and $\mathrm{SHH}$. Aberrant activation of these pathways alters the expression of various critical target genes mediating the processes of apoptosis, proliferation and angiogenesis. To further elucidate the mechanisms of antitumor activity of NC, we determined its effect on the activation of STAT3, ERK and SHH pathways. Activation of STAT3 and ERK is mediated by its phosphorylation, we therefore investigated STAT3 and ERK activation in HCC tumor tissues and/or HepG2 cells by Western Blot analysis using antibodies that recognize STAT3 or ERK phosphorylation. As shown in Figure 8A and B, NC treatment decreased the levels of phosphorylated STAT3 and ERK in tumors of xenograft mice and/or in HepG2 cells. The levels of non-phosphorylated STAT3 and ERK remained unchanged. To further explore the mechanisms whereby NC suppressed the activation of STAT3, we performed Dual Luciferase Reporter assay to examine STAT3 transcriptional activity in HepG2 cells. Results from Figure $8 \mathrm{C}$ showed that NC significantly and dosedependently inhibited IL-6-stimulated increase of STAT3 transcriptional activity. We assessed the effect of $\mathrm{NC}$ on the expression of key mediators of SHH pathway in hepatic cancer xenograft tumors and HepG2 cells using IHC, Western Blot and RT-PCR analyses. As shown in Figure $9 \mathrm{~A}$ and $\mathrm{B}, \mathrm{NC}$ treatment significantly decreased the protein expression of SHH- or Gli-1-positive cells both in the hepatic cancer xenograft tumors and HepG2 cells. Data from RT-PCR showed that the pattern of mRNA expression was similar to their respective protein levels (Figure $9 \mathrm{C}$ and $\mathrm{D}$ ). Taken together, these data suggest that NC significantly suppresses the activation of multiple hepatic cancer-related signaling pathways.

\section{Conclusions}

In summary, here for the first time we demonstrate that Nitidine chloride possesses a broad range of anti-cancer activities due to its ability to affect multiple intracellular targets. Our findings suggest that NC could be a novel therapeutic agent for the treatment of hepatic cancer and other malignancies. However, it remains unclear how NC interacts with STAT3, ERK or hedgehog signaling and deactivate it, although we demonstrate these multiple signaling pathways are affected by NC. It is unknown whether $\mathrm{NC}$ is a direct transcriptional suppressor of STAT3, ERK, SHH or Gli. In addition, the dose of NC used in in vitro study is as high as $50 \mu \mathrm{M}$. The concentration of NC certainly should be much lower if we try to demonstrate its druggability in pharmaceutical research. These intriguing questions must be addressed in future studies before NC can be further developed as a multi-target drug for cancer therapy.

\section{Competing interest}

The authors declare that they have no competing interests.

\section{Authors' contributions}

Study concept and design: JM Lin, J Peng; Acquisition of data: JM Lin, AL shen, HW Chen, J Liao, T Xu, LY Liu, J Lin; Analysis and Interpretation of Data: JM Lin, J Peng; Drafting of the manuscript: JM Lin, J Peng; Statistical analysis: JM Lin, J Peng; Technical and material support: JM Lin, AL shen, HW Chen, J Liao, T Xu, LY Liu, J Lin, J Peng. All authors have read and approved the final manuscript.

\section{Acknowledgments}

This work was sponsored by the National Natural Science Foundation of China (81073097 and 81001554).

\section{Author details}

${ }^{1}$ Academy of Integrative Medicine Biomedical Research Center, Fujian University of Traditional Chinese Medicine, Fuzhou, Fujian 350122, China. ${ }^{2}$ Fujian Key Laboratory of Integrative Medicine on Geriatrics, Fujian University of Traditional Chinese Medicine, Fuzhou, Fujian 350122, China. ${ }^{3}$ Department of Acupuncture and Moxa and Tuina, Fujian University of Traditional Chinese Medicine, Fuzhou, Fujian 350122, China.

Received: 31 March 2014 Accepted: 26 September 2014

Published: 30 September 2014

\section{References}

1. Gomaa Al, Khan SA, Toledano MB, Waked I, Taylor-Robinson SD: Hepatocellular carcinoma: Epidemiology, risk factors and pathogenesis. World J Gastroenterol 2008, 14:4300-4308.

2. Montalto G, Cervello M, Giannitrapani L, Dantona F, Terranova A, Castagnetta LA: Epidemiology, risk factors, and natural history of hepatocellular carcinoma. Ann N Y Acad Sci 2003, 963:13-20.

3. Sherman M: Hepatocellular carcinoma: epidemiology, risk factors, and screening. Semin Liver Dis 2005, 25:143-154.

4. Jemal A, Bray F, Center MM, Ferlay J, Ward E, Forman D: Global cancer statistics. CA Cancer J Clin 2011, 61:69-90.

5. Yeh CT, Chen TC, Chang ML, Hsu CW, Yeh TS, Lee WC, Huang SF, Tsai CC: Identification of NV-F virus DNA in hepatocellular carcinoma. J Med Virol 2007, 79:92-96.

6. Levin B, Amos C: Therapy of unresectable hepatocellular carcinoma. N Engl J Med 1995, 332:1294-1296.

7. Abou-Alfa GK, Huitzil-Melendez FD, O'Reilly EM, Saltz LB: Current management of advanced hepatocellular carcinoma. Gastrointest Cancer Res 2008, 2:64-70.

8. Boose G, Stopper H: Genotoxicity of several clinically used topoisomerase II inhibitors. Toxicol Lett 2000, 116:7-16.

9. Aggarwal BB, Kunnumakkara AB, Harikumar KB, Gupta SR, Tharakan ST, Koca C, Dey S, Sung B: Signal transducer and activator of transcription-3, inflammation, and cancer: how intimate is the relationship? Ann NY Acad Sci 2009, 1171:59-76.

10. Bromberg J, Wang TC: Inflammation and Cancer: IL-6 and STAT3 Complete the Link. Cancer Cell 2009, 15:79-80. 
11. Kusaba T, Nakayama T, Yamazumi K, Yakata Y, Yoshizaki A, Inoue K, Nagayasu T, Sekine I: Activation of STAT3 is a marker of poor prognosis in human colorectal cancer. Oncol Rep 2006, 15:1445-1451.

12. Theunissen JW, de Sauvage FJ: Paracrine hedgehog signaling in cancer. Cancer Res 2009, 69:6007-6010.

13. Mazumdar T, DeVecchio J, Shi T, Jones J, Agyeman A, Houghton JA Hedgehog signaling drives cellular survival in human colon carcinoma cells. Cancer Res 2011, 71:1092-1102.

14. Das S, Tucker JA, Khullar S, Samant RS, Shevde LA: Hedgehog signaling in tumor cells facilitates osteoblast-enhanced osteolytic metastases. PLoS One 2012, 7:e34374

15. Varjosalo M, Taipale J: Hedgehog: functions and mechanisms. Genes Dev 2008, 22:2454-2472

16. Claret S, Sanial M, Plessis A: Evidence for a novel feedback loop in the hedgehog pathway involving smoothened and fused. Curr Biol 2007, 17:1326-1333

17. Wei L, Lin J, Xu W, Cai Q, Shen A, Hong Z, Peng J: Scutellaria barbata D. Don inhibits tumor angiogenesis via suppression of hedgehog pathway in a mouse model of colorectal cancer. Int J Mol Sci 2012, 13:9419-9430.

18. Seger R, Krebs EG: The MAPK signaling cascade. FASEB J 1995, 9:726-735.

19. Roberts PJ, Der CJ: Targeting the Raf-MEK-ERK mitogen-activated protein kinase cascade for the treatment of cancer. Oncogene 2007, 26:3291-3310.

20. Giuliani N, Lunghi P, Morandi F, Colla S, Bonomini S, Hojden M, Rizzoli V, Bonati A: Downmodulation of ERK protein kinase activity inhibits VEGF secretion by human myeloma cells and myeloma-induced angiogenesis. Leukemia 2004, 18:628-635.

21. Gordaliza M: Natural products as leads to anticancer drugs. Clin Trans/ Oncol 2007, 9:767-776.

22. Newman DJ, Cragg GM, Snader KM: The influence of natural products upon drug discovery. Nat Prod Rep 2000, 17:215-234.

23. Del Poeta M, Chen SF, Von Hoff D, Dykstra CC, Wani MC, Manikumar G, Heitman J, Wall ME, Perfect JR: Comparison of in vitro activities of camptothecin and nitidine derivatives against fungal and cancer cells. Antimicrob Agents Chemother 1999, 43:2862-2868.

24. Hu J, Zhang WD, Liu RH, Zhang C, Shen YH, Li HL, Liang MJ, Xu XK: Benzophenanthridine alkaloids from Zanthoxylum nitidum (Roxb.) DC, and their analgesic and anti-inflammatory activities. Chem Biodivers 2006, 3:990-995.

25. Iwasaki H, Okabe T, Takara K, Toda T, Shimatani M, Oku H: Tumorselective cytotoxicity of benzo [c] phenanthridine derivatives from Toddalia asiatica Lam. Cancer Chemother Pharmacol 2010, 65:719-726.

26. Chen J, Wang J, Lin L, He L, Wu Y, Zhang L, Yi Z, Chen Y, Pang X, Liu M: Inhibition of STAT3 signaling pathway by nitidine chloride suppressed the angiogenesis and growth of human gastric cancer. Mol Cancer Ther 2012, 11:277-287.

27. Liao J, Xu T, Zheng JX, Lin JM, Cai QY, Yu DB, Peng J: Nitidine chloride inhibits hepatocellular carcinoma cell growth in vivo through the suppression of the JAK1/STAT3 signaling pathway. Int J Mol Med 2013, 32:79-84.

28. Nurse P: Ordering S phase and M phase in the cell cycle. Cell 1994 79:547.

29. Morgan DO: Principles of CDK regulation. Nature 1995, 374:131.

30. Harakeh S, Abu-El-Ardat K, Diab-Assaf M, Niedzwiecki A, El-Sabban M, Rath M: Epigallocatechin-3-gallate induces apoptosis and cell cycle arrest in HTLV-1-positive and-negative leukemia cells. Med Oncol 2008, 25:30-39.

31. Kessel D, Luo Y: Cells in cryptophycin-induced cell-cycle arrest are susceptible to apoptosis. Cancer Lett 2000, 151:25-29.

32. Purohit A, Hejaz HA, Walden L, MacCarthy-Morrogh L, Packham G, Potter BV, Reed MJ: The effect of 2-methoxyoestrone-3-O-sulphamate on the growth of breast cancer cells and induced mammary tumours. Int $J$ Cancer 2000, 85:584-589.

33. Youle RJ, Strasser A: The BCL-2 protein family: opposing activities that mediate cell death. Nat Rev Mol Cell Biol 2008, 9:47-59.

34. Folkman J: Tumor angiogenesis: therapeutic implications. N Engl J Med 1971, 285:1182-1186.

35. Folkman J: Angiogenesis in cancer, vascular, rheumatoid and other diseases. Nat Med 1995, 1:27-31.

36. Folkman J: Angiogenesis. Annu Rev Med 2006, 57:1-18.

37. Cook KM, Figg WD: Angiogenesis inhibitors: current strategies and future prospects. CA Cancer J Clin 2010, 60:222-243.
38. Folkman J: How is blood vessel growth regulated in normal and neoplastic tissue? G.H.A. Clowes memorial award lecture. Cancer Res 1986, 46:467-473.

39. Jain RK: Transport of molecules in the tumor interstitium: a review. Cancer Res 1987, 47:3039-3051.

40. Kerbel RS: Tumor angiogenesis. N Engl J Med 2008, 358:2039-2049.

41. Dai J, Wan S, Zhou F, Myers RE, Guo X, Li B, Fu X, Palazzo JP, Dou K, Yang H, Xing J: Genetic polymorphism in a VEGF-independent angiogenesis gene ANGPT1 and overall survival of colorectal cancer patients after surgical resection. PLoS One 2012, 7:e34758

42. Martins SF, Garcia EA, Luz MA, Pardal F, Rodrigues M, Filho AL: Clinicopathological correlation and prognostic significance of VEGF-A VEGF-C, VEGFR-2 and VEGFR-3 expression in colorectal cancer. Cancer Genomics Proteomics 2013, 10:55-67.

43. Ferrara N, Gerber HP, LeCouter J: The biology of VEGF and its receptors. Nat Med 2003, 9:669-676.

44. Gille H, Kowalski J, Li B, LeCouter J, Moffat B, Zioncheck TF, Pelletier N, Ferrara $\mathrm{N}$ : Analysis of biological effects and signaling properties of Flt-1 (VEGFR-1) and KDR (VEGFR-2). A reassessment using novel receptor-specific vascular endothelial growth factor mutants. J Bio/ Chem 2001, 276:3222-3230.

45. Kaya M, Wada T, Akatsuka T, Kawaguchi S, Nagoya S, Shindoh M, Higashino F, Mezawa F, Okada F, Ishii S: Vascular endothelial growth factor expression in untreated osteosarcoma is predictive of pulmonary metastasis and poor prognosis. Clin Cancer Res 2000, 6:572-577.

doi:10.1186/1471-2407-14-729

Cite this article as: Lin et al:: Nitidine chloride inhibits hepatic cance growth via modulation of multiple signaling pathways. BMC Cancer $201414: 729$

\section{Submit your next manuscript to BioMed Central and take full advantage of:}

- Convenient online submission

- Thorough peer review

- No space constraints or color figure charges

- Immediate publication on acceptance

- Inclusion in PubMed, CAS, Scopus and Google Scholar

- Research which is freely available for redistribution 